\title{
Dependence of tissue inhomogeneity correction factors on photon-beam \\ energy
}

Md. Akhtaruzzaman, Pawel Kukolowicz

\begin{abstract}
Introduction: Commissioning of the treatment-planning system includes the accuracy of dose calculations in the inhomogeneous absorber. Several results of measurements with regard to inhomogeneity correction factors (CFs) have been published. However, the dependence of CFs on photon-beam energy may preclude such results from being applied to the photon beams of general users. Purpose: The aim of this study was to assess the dependence of CFs on the photon-beam energy. Materials and methods: CFs were calculated by the Batho method for several slab geometries comprised of concentrations of lung tissue and water of $0.25 \mathrm{and} 1.00 \mathrm{~g} / \mathrm{cm}^{3}$, respectively. The CFs were calculated at $6 \mathrm{MV}\left(\mathrm{TPR}_{10}^{20}=0.67 \pm k * 0.01\right)$ and $15 \mathrm{MV}\left(\mathrm{TPR}_{10}^{20}=0.76 \pm k * 0.01\right)$ where $k=-3,-2,-1,0,1,2,3$. All calculations were performed in the region where a charged-particle equilibrium exists. Results: Changes in CFs of less than $2 \%$ were observed across the considered energy ranges. With a change in $\mathrm{TPR}_{20,10}$ of 0.01 , both at 6 and $15 \mathrm{MV}$ at a depth of $5 \mathrm{~cm}$ below the lung; and lung thicknesses of 3, 5 and $8 \mathrm{~cm}$ over a field surface area of $10 \times 10 \mathrm{~cm}^{2}$, the change in CF never exceeded $2.4 \%$. The dependences of changes in CFs in terms of $\mathrm{TPR}_{20,10}$ were $1.74 \%$ and $1.20 \%$ for field surface areas of $5 \times 5 \mathrm{~cm}^{2}$ and $20 \times 20 \mathrm{~cm}^{2}$, respectively. A comparison of 42 linear accelerators (LINACs) exhibiting 6 MV and 15 MV of energy installed in Poland showed that the maximum differences in terms of $\mathrm{TPR}_{20,10}$ at $6 \mathrm{MV}$ and $15 \mathrm{MV}$ were $4.2 \%$ and $2.2 \%$, respectively. Conclusion: A linear dependence of CFs on energy was observed. According to observations, the smaller the surface area of the field and deeper the point of interest below the lung, the more dependent CFs are on energy.
\end{abstract}

Keywords: Batho method $\bullet$ dose calculation $\bullet$ inhomogeneity correction factors $\bullet$ photon-beam energy

\author{
Md. Akhtaruzzaman ${ }^{凶}$ \\ Faculty of Physics \\ University of Warsaw \\ Ludwika Pasteura 5, 02-093 Warsaw, Poland \\ and Department of Medical Physics \\ Maria Skłodowska-Curie Memorial Cancer Centre and \\ Institute of Oncology \\ Roentgena 5, 02-781 Warsaw, Poland \\ E-mail: akh_zam@yahoo.com \\ P. Kukolowicz \\ Department of Medical Physics \\ Maria Skłodowska-Curie Memorial Cancer Centre and \\ Institute of Oncology \\ Roentgena 5, 02-781 Warsaw, Poland
}

Received: 24 January 2017

Accepted: 19 December 2017

\section{Introduction}

The human body is comprised of various tissues and cavities that possess different physical and radiological properties. From a radiation dosimetry perspective, the most significant tissues and cavities are radiologically different from water, for example, the lungs, oral cavities, teeth, nasal passages, sinuses and bones. In some cases, foreign materials such as metallic prostheses are also present. To maximize the therapeutic benefit of radiation therapy, it is essential that the absorbed dose should be delivered accurately to all the irradiated tissues in the presence of such inhomogeneity [1].

The treatment-planning process is one of the most important steps in the preparation of radiotherapy treatment. The aim of treatment planning is to choose the best energy intensity and number of treatment beams - their directions, intensities and modulations - to conform with the dose distribution to the target and minimize the dose delivered to normal structures as much as feasibly possible. The 
credibility of the treatment plan depends on the accuracy of dose-distribution calculations. In order to increase their accuracy, dose-calculation models are being implemented in treatment-planning systems [2-14]. Whatever dose-distribution-calculation algorithm is used, before its first clinical application the accuracy of dose-distribution calculations has to be verified. From the user's point of view, the verification of the calculations performed in inhomogeneous media is challenging. One of the tools most often used is to compare the calculated and measured so-called correction factors (CFs) for inhomogeneities. The correction factor is defined as the ratio of the dose in a heterogeneous medium to the dose at the same point in a homogeneous water-like medium.

Measuring correction factors is time-consuming and resource-intensive. In the literature, results of such investigations can be found $[15,16]$. For the sake of simplicity, CFs are usually measured for slab geometries. It would be very helpful if the CFs measured by one user could be used by another. However, it is uncertain whether data taken from the literature are applicable to actual situations. In other words, the question arises of how much the user's beam differs from the one which had been used for the measurement of correction factors. Two photon beams of $6 \mathrm{MV}$ and $15 \mathrm{MV}$ "nominal" energies are used most often, though beams of the same nominal energy may exhibit differing photon-energy spectra. In this study, the dependence of inhomogeneity correction factors on energy in terms of $6 \mathrm{MV}$ and $15 \mathrm{MV}$ photon beams was investigated. Correction factors were calculated by the Batho method [17], which for many years has been used in modern treatment-planning systems. To calculate the correction factors according to the Batho method, tissue air ratios (TARs) were generated using the formulae for percentage depth dose (PDD) proposed by Gerbi [18] and peak scatter factors (PSFs) according to the method described by $\mathrm{Li}$ [19]. More details concerning the ideas of Gerbi and Li may be found in the next paragraph.

\section{Methods and materials}

To obtain the dependence of the correction factors on actual intensities of energy, CFs were calculated using the Batho power law method. According to Batho [17], the correction factor is given as the ratio of powers of TARs. For a layer geometry as shown in Fig. 1, the correction factor is described by the formula:

$$
\mathrm{CF}=\frac{T\left(d_{1}, A\right)^{\rho_{a}-\rho_{b}}}{T\left(d_{2}, A\right)^{1-\rho_{b}}}
$$

where: $T$ - tissue air ratio at depths $d_{1}$ or $d_{2}$ of field size $A ; \rho_{a}$ - density of the material in which point $\mathrm{P}$ lies at a depth $d_{1}$ below its surface; $\rho_{b}$ - density of an overlying material of thickness $\left(d_{2}-d_{1}\right)$.

In this work, the tissue air ratios (TARs) were calculated using the following formula:

$$
\text { (2) } \begin{aligned}
T\left(A \frac{R+d}{I}\right)= & \frac{(R+d)^{2}}{\left(R+d_{\max }\right)^{2}} \cdot \operatorname{PSF}\left(A \frac{R+d_{\max }}{I}\right) \\
& \cdot \operatorname{PDD}(A, d, R)
\end{aligned}
$$

where: $A$ - field size, $d$ - depth, $R$ - source-to-surface distance, $I$ - source-to-isocenter distance, PDD percentage depth dose.

The PDDs were described by the formulae proposed by Gerbi [18]:

$$
\mathrm{PDD}=\log ^{-1}\left[p_{1}+p_{2} d+\left(p_{3}+p_{4} d\right) \log \frac{A}{P}\right]
$$

where: $P$ - perimeter, $d$ - depth, $p_{1}$ through $p_{4}$ are approximated by:

$$
\begin{aligned}
& p_{1}=q_{1}+q_{2} * \mathrm{TPR}_{20,10}+q_{3}\left(\mathrm{TPR}_{20,10}\right)^{2}, \\
& p_{2}=q_{4}+q_{5} * \mathrm{TPR}_{20,10}, \\
& p_{3}=q_{6}+q_{7} * \mathrm{TPR}_{20,10}, \\
& p_{4}=q_{8}+q_{9} * \mathrm{TPR}_{20,10}, \\
& q_{1}=1.993418, q_{2}=-0.057964, q_{3}=0.223669, \\
& q_{4}=-0.071951, q_{5}=0.067835, q_{6}=0.038277, \\
& q_{7}=-0.074943, q_{8}=0.030567, q_{9}=-0.034585 .
\end{aligned}
$$

The peak scatter factors (PSF) were calculated using the formula proposed by $\mathrm{Li}$ [19]:

$$
\mathrm{PSF}=1+\frac{m A}{A+n}
$$

where: $m=0.1090$ (6 MV), $0.0917(15 \mathrm{MV})$; $n=4.9173(6 \mathrm{MV}), 3.8347$ (15 MV); $A$ = field size.

CFs were calculated for several energies of photon beams described in terms of the so-called Quality Index - TPR 20.10 .

$\left(\mathrm{TPR}_{10}^{20}=0.67 \pm k * 0.01\right)$ for $6 \mathrm{MV}, k=-3,-2$, $-1,0,1,2,3$,

$\left(\mathrm{TPR}_{10}^{20}=0.76 \pm k * 0.01\right)$ for $15 \mathrm{MV}, k=-3$, $-2,-1,0,1,2,3$.

$\mathrm{TPR}_{20,10}$ of 0.67 and 0.76 are typical values of the Quality Index for the nominal photon energies of $6 \mathrm{MV}$ and $15 \mathrm{MV}$, respectively.

CFs were calculated for the cases which mimic irradiation in the thorax region, i.e. for a lung of $0.25 \mathrm{~g} / \mathrm{cm}^{3}$ in density and for field sizes of $5 \times 5$, $10 \times 10,15 \times 15$ and $20 \times 20 \mathrm{~cm}^{2}$. The build-up thickness $\left(d_{3}\right)$ was kept constant at $3 \mathrm{~cm}$ (Fig. 1). Depths of 2, 5, 8 and $10 \mathrm{~cm}, 5 \mathrm{~cm}$ below the lung and at a depth of $5 \mathrm{~cm}$ in terms of three different thicknesses of the lung of 3,5 and $8 \mathrm{~cm}$ were considered

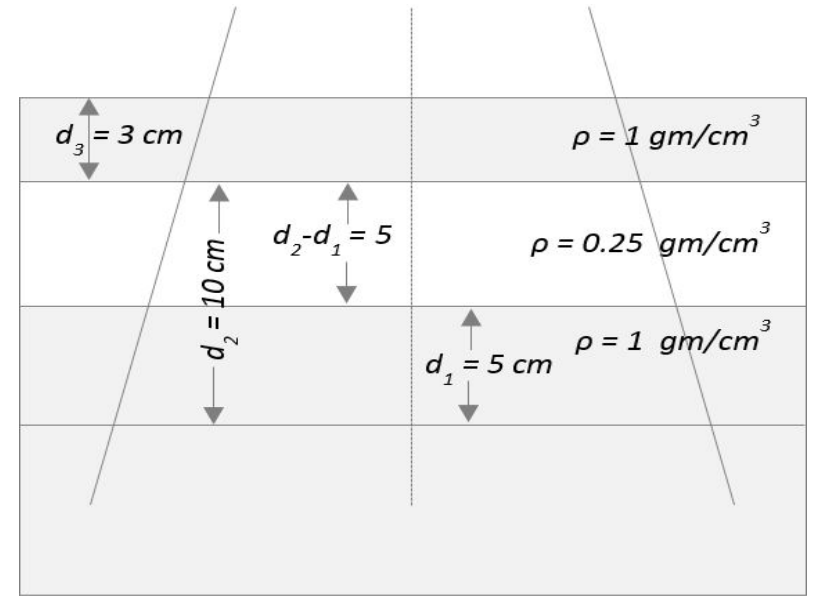

Fig. 1. The geometry for which the CFs were calculated. 


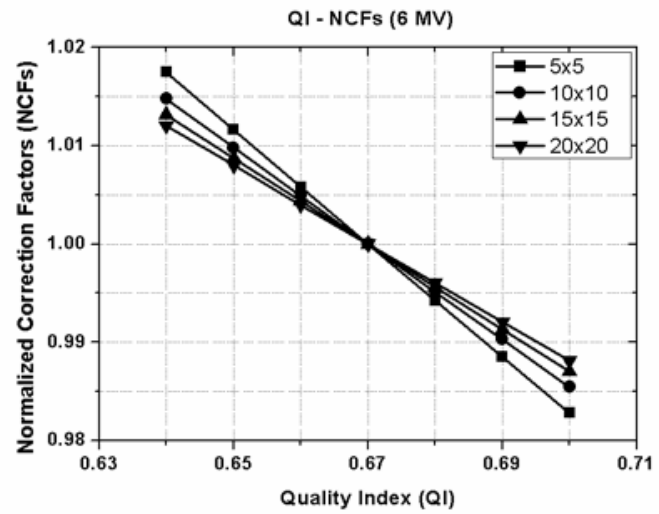

b

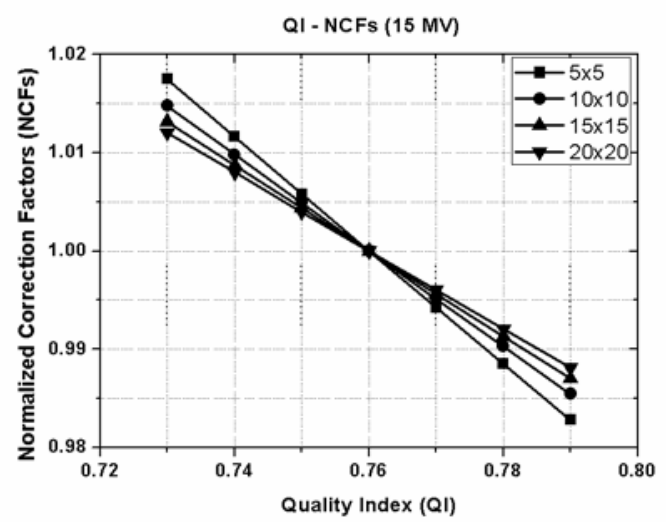

Fig. 2. Normalized correction factors as a function of beam quality index at a depth of $5 \mathrm{~cm}, 5 \mathrm{~cm}$ below the lung for $6 \mathrm{MV}(\mathrm{a})$ and $15 \mathrm{MV}$ (b) X-rays with field sizes of $5 \times 5,10 \times 10,15 \times 15$ and $20 \times 20 \mathrm{~cm}^{2}$.

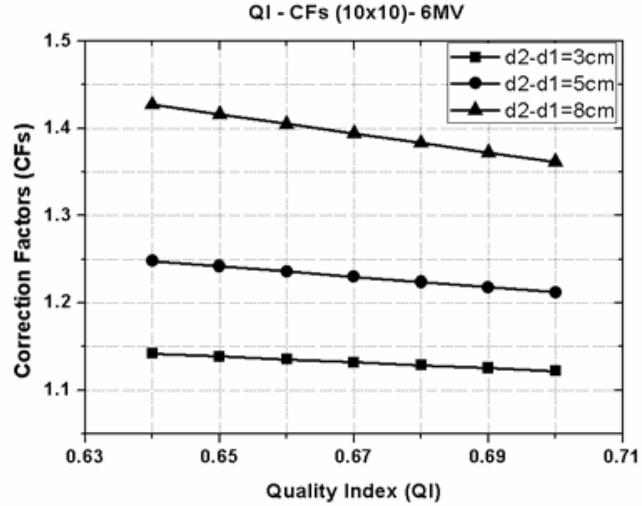

$\mathrm{b}$

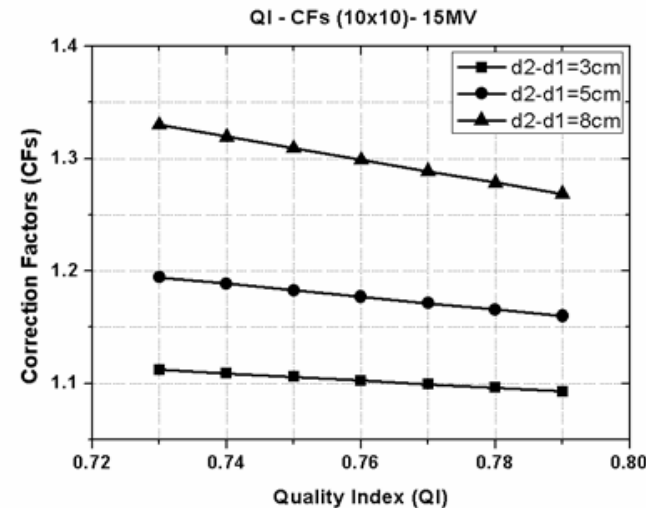

Fig. 3. Correction factors as a function of beam quality index at a depth of $5 \mathrm{~cm}, 3,5$ and $8 \mathrm{~cm}$ below the lung for $6 \mathrm{MV}$ (a) and $15 \mathrm{MV}$ (b) X-rays with a field size of $10 \times 10 \mathrm{~cm}^{2}$.

in the calculations which were all performed by the Visual Basic program.

\section{Results}

Figure 2 exhibited the linear dependence of CFs on energy for four different beam sizes calculated at a depth of $5 \mathrm{~cm}, 5 \mathrm{~cm}$ below the lung and changes in CFs of up to $2 \%$ were observed across the considered energy ranges. The dependence of CFs on energy is demonstrated for three different thicknesses of lung $(3,5$ and $8 \mathrm{~cm})$ over a field size of $10 \times 10 \mathrm{~cm}^{2}$ in
Fig. 3. The results yielded a maximum difference of $2.4 \%$. Figure 4 also show the linear dependence of the CFs on energy at four different depths $(2,5,8$ and $10 \mathrm{~cm}$ ) at $5 \mathrm{~cm}$ below the lung over a field size of $10 \times 10 \mathrm{~cm}^{2}$ and the largest difference of $1.5 \%$ was found at a depth of $10 \mathrm{~cm}$.

\section{Discussion}

The dose distribution for each treatment plan is calculated by a mathematical model implemented in the treatment-planning system. The accuracy

$\mathrm{b}$

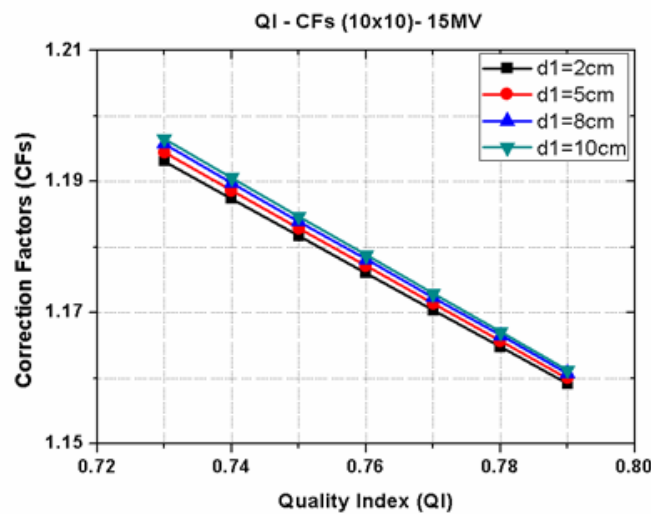

Fig. 4. Correction factors as a function of beam quality index at depths of 2, 5, 8 and $10 \mathrm{~cm}, 5 \mathrm{~cm}$ below the lung for $6 \mathrm{MV}$ (a) and $15 \mathrm{MV}$ (b) X-rays with a field size of $10 \times 10 \mathrm{~cm}^{2}$. 
of dose distribution calculations is always limited, therefore, before the first clinical application of each treatment planning system (TPS), the accuracy of dose-calculation algorithms has to be verified. International recommendations propose that several quality-control tests should be performed for treatment-planning systems. Some of them are based on a comparison of the measured and calculated dose distributions [20-26]. These comparisons can be quite easily drawn if the dose distributions are measured and calculated in a homogeneous water-like phantom substance. The results of such comparisons yield very good agreement for most of the treatment-planning systems used today. The worst results are obtained in heterogeneous anatomies, especially if the lungs are present. From the user's point of view, it would be very helpful to have a set of correction factors that were calculated for well-described inhomogeneous situations. Two nominal energies, namely $6 \mathrm{MV}$ and $15 \mathrm{MV}$, are used most frequently in clinical practice. Unfortunately, the same nominal energy does not yield the same actual energy nor the same spectrum of energy. There are some differences which are fortunately not very large. In this work, CFs for lung inhomogeneities were intentionally calculated for the same nominal energies, namely $6 \mathrm{MV}$ and $15 \mathrm{MV}$, but different actual energies - different Quality Indexes.

The study showed that there was a linear dependence of correction factors on energy. The higher the energy, the lower the CF. This dependence is more pronounced if the thickness of the lung is higher and the calculation point is deeper below the lung. A change in the $\mathrm{TPR}_{20,10}$ of 0.01 - both for energies of 6 and $15 \mathrm{MV}$ - at a depth of $5 \mathrm{~cm}$ below the lung, and lung thicknesses of 3,5 and $8 \mathrm{~cm}$ leads to a change in CF of $0.87,1.45$ and $2.38 \%$, respectively. In addition, the size of the field influences these dependencies. The smaller the field size, the larger the influence of changes in energy on the CFs. For a $5 \times$ $5 \mathrm{~cm}^{2}$ field size, a change in the TPR 20,10 of 0.01 leads to a change in the CF of $1.74 \%$, but for a field size of $20 \times 20 \mathrm{~cm}^{2}$, the change in the CF is of only $1.2 \%$.

The comparison was performed for a nominal energy of $6 \mathrm{MV}$ when $\mathrm{TPR}_{20,10}=0.67 \pm 6 \%$, and for a nominal energy of $15 \mathrm{MV}$ when $\mathrm{TPR}_{20,10}=0.76 \pm 6 \%$. To obtain more detailed results of how the $\mathrm{TPR}_{20,10}$ differs for linear accelerators of the same nominal energy, data from 21 linear accelerators (linacs) with a nominal energy of $6 \mathrm{MV}$, and 21 linear accelerators (linacs) with a nominal energy of $15 \mathrm{MV}$ were collected from linacs installed in Poland (Fig. 5). The maximum differences in $\mathrm{TPR}_{20,10}$ were found to be $4.2 \%$ and $2.2 \%$ for nominal energies of $6 \mathrm{MV}$ and $15 \mathrm{MV}$, respectively. Therefore, it may be concluded that our results are applicable for all accelerators installed in Poland.

To calculate the TARs, calculated PDDs were used derived from a formula proposed by Gerbi. This formula is not applicable for field sizes less that $4 \times 4 \mathrm{~cm}^{2}$ and at depths shallower than $d_{\max }+1 \mathrm{~cm}$ or greater than $30 \mathrm{~cm} \mathrm{[18].} \mathrm{To} \mathrm{calculate} \mathrm{the} \mathrm{correc-}$ tion factors, the Batho power law method was used. This method has been considered the best available

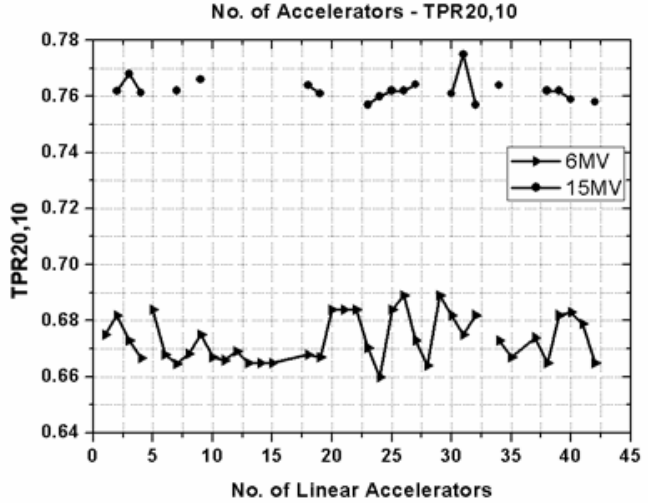

Fig. 5. $\mathrm{TPR}_{20,10}$ as a function of different linear accelerators (linacs) for 6 and 15 MV X-rays.

for several years. There are several papers in which authors have analysed the accuracy of the Batho power law method [27-33] which showed that the method yields very good results for geometries in which a charge-particle equilibrium and slab geometry exist. An improvement in the accuracy of dose calculations at points within a lung of as much as $5 \%$ can be achieved simply by using the Batho power law method. This is particularly important for larger field sizes where the scattered dose plays an important role. However, due to tremendous developments in terms of the calculation of algorithms with regard to treatment planning systems, this method is not widely used nowadays since it does not take into account electron transport. Electron transport is important in regions where no charged-particle equilibrium exists. Therefore, this paper is limited to regions where electric charged-particle equilibria are present. In terms of these cases, other methods that are more sophisticated have to be applied. This problem will be addressed in our next work.

\section{Conclusions}

The linear dependence of correction factors on photon-beam energy was investigated. Correction factors decreased with an increase in the size of the field. The thicker the lung is, the larger the differences between the correction factors are. Also, as the depth below a lung increases, so does the correction factor. To our knowledge, this is the first published study demonstrating the dependence of inhomogeneity correction factors on the quality of photon beams. These factors could be utilized by readers concerned with the quality control of treatment-planning systems.

\section{References}

1. Papanikolaou, N., Battista, J. J., Boyer, A. L., Kappas, C., Klein, E., Mackie, T. R., Sharpe, M., \& Van Dyk, J. (2004). Tissue inhomogeneity corrections for megavoltage photon beams. Madison WI: Medical Physics Publishing. (AAPM Report No. 85).

2. Das, I. J., Ding, G. X., \& Ahnesjö, A. (2008). Small fields: Non-equilibrium radiation dosimetry. Med. Phys., 35(1), 206-215. DOI: 10.1118/1.2815356. 
3. Robinson, D. (2008). Inhomogeneity correction and the analytic anisotropic algorithm. J. Appl. Clin. Med. Phys., 9(2), 112-122.

4. Ding, W., Johnston, P. N., Wong, T. P. Y., \& Bubb, I. F. (2004). Investigation of photon beam models in heterogeneous media of modern radiotherapy. Australas Phys. Eng. Sci., 27, 39-48. DOI: 10.1007/ BF03178375.

5. Carrasco, P., Jornet, N., Duch, M., Weber, L., Ginjaume, M., Endaldo, T., Jurado, D., Ruiz, A., \& Ribas, M. (2004). Comparison of dose calculation algorithms in phantoms with lung equivalent heterogeneities under conditions of lateral electronic disequilibrium. Med. Phys., 31, 2899-2911. DOI: 10.1118/1.1788932.

6. Krieger, T., \& Sauer, O. A. (2005). Monte Carlo versus pencil-beam-/collapsed-cone-dose calculation in a heterogeneous multi-layer phantom. Phys. Med. Biol., 50(5), 859-868. DOI: 10.1088/0031-9155/50/5/010.

7. Van Esch, A., Tillikainen, L., Pyykkonen, J., Tenhunen, M., Helminen, H., Siljamaki, S., Alakuijala, J., Paiusco, M., Iori, M., \& Huyskens, D. (2006). Testing of the analytical anisotropic algorithm for photon dose calculation. Med. Phys., 33(11), 4130-4148. DOI: 10.1118/1.2358333.

8. Dobler, B., Walter, C., Knopf, A., Fabri, D., Loeschel, R., Polednik, M., Schneider, F., Wenz, F., \& Lohr, F. (2006). Optimization of extracranial stereotactic radiation therapy of small lung lesions using accurate dose calculation algorithms. Radiat. Oncol., 1, 45(11pp.). DOI: 10.1186/1748-717X-1-45.

9. Vanderstraeten, B., Reynaert, N., Paelinck, L., Madani, I., De Wagter, C., De Gersem, W., De Neve, W., \& Thierens, H. (2006). Accuracy of patient dose calculation for lung IMRT: A comparison of Monte Carlo, convolution/superposition and pencil beam computations. Med. Phys., 33(9), 3149-3158. DOI: 10.1118/1.2241992.

10. Gray, A., Oliver, L. D., \& Johnston, P. N. (2009). The accuracy of the pencil beam convolution and anisotropic analytical algorithms in predicting the dose effects due to attenuation from immobilization devices large air gaps. Med. Phys., 36(7), 3181-3191. DOI: 10.1118/1.3147204.

11. Rana, S., Rogers, K., Lee, T., Reed, D., \& Biggs, C. (2013). Verification and dosimetric impact of Acuros XB algorithm for stereotactic body radiation therapy (SBRT) and RapidArc planning for non-small-cell lung (NSCLC) patients. Int. J. Med. Phys. Clin. Eng. Radiat. Oncol., 2(1), 6-14. DOI: 10.4236/ ijmpcero.2013.21002.

12. Han, T., Mourtada, F., Kisling, K., Mikell, J., Followill, D., \& Howell, R. (2012). Experimental validation of deterministic Acuros XB algorithm for IMRT and VMAT dose calculations with the Radiological Physics Center's head and neck phantom. Med. Phys., 39(4), 2193-2202. DOI: 10.1118/1.3692180.

13. Rana, S., \& Rogers, K. (2013). Dosimetric evaluation of Acuros XB dose calculation algorithm with measurements in ness for smaller and larger field sizes. Med. Phys., 38, 9-14.

14. Stephen, O. (2013). Dose prediction accuracy of collapsed cone convolution superposition algorithm in a multi-layer inhomogenous phantom. Int. J. Cancer Ther. Oncol., 1(1), 01016(4pp.). DOI: 10.14319/ ijcto.0101.6.

15. El-Khatib, E. E., Evans, M., Pla, M., \& Cunningham, J. R. (1989). Evaluation of lung dose correction methods for photon irradiations of thorax phantoms. Int. J. Radiat. Oncol. Biol. Phys., 17, 871-878.
16. Orton, C. G., Chungbin, S., \& Klein, E. E., Gillin, M T., Schultheiss, T. E., \& Sanse, W. T. (1998). Study of lung density corrections in a clinical trial (RTOG 88-08). Radiation Therapy Oncology Group. Int. J. Radiat. Oncol. Biol. Phys., 41(4), 787-794. DOI: 10.1016/S0360-3016(98)00117-5.

17. Batho, H. F. (1964). Lung corrections in cobalt 60 beam therapy. J. Can. Assoc. Radiol., 15, 79-83.

18. Gerbi, B. J. (1991). A mathematical expression for \%DD accurate from Co-60 to 24 MV. Med. Phys., 18(4), 724-726. DOI: 10.1118/1.596666.

19. Li, X. A. (1999). Peak scatter factors for high-energy photon beams. Med. Phys., 26(6), 962-966. DOI 10.1118/1.598489.

20. ICRU. (1987). Use of computers in external beam radiotherapy procedures with high-energy photons and electrons. Maryland: ICRU Publications. (ICRU Report No. 42).

21. Ekstrand, K. E., \& Barnes, W. H. (1990). Pitfalls in the use of high energy $\mathrm{X}$ rays to treat tumors in the lung. Int. J. Radiat. Oncol. Biol. Phys., 18(1), 249-252.

22. Hunt, M. A., Desobry, G. E., Fowble, B., \& Coia, L. R. (1997). Effect of low-density lateral interfaces on soft-tissue doses. Int. J. Radiat. Oncol. Biol. Phys., 37(2), 475-482.

23. Kornelsen, R. O., \& Young, M. E. (1982). Changes in the dose-profile of a $10 \mathrm{MV}$ x-ray beam within and beyond low-density material. Med. Phys., 9, 114-116. DOI: 10.1118/1.595059.

24. Rice, R. K., Mijnheer, B. J., \& Chin, L. M. (1988). Benchmark measurements for lung dose corrections for X-ray beams. Int. J. Radiat. Oncol. Biol. Phys., 15(2), 399-409. DOI: 10.1016/S03603016(98)90022-0.

25. Yorke, E., Harisiadis, L., Wessels, B., Aghdam, H., \& Altemus, R. (1996). Dosimetric considerations in radiation therapy of coin lesions of the lung. Int. J. Radiat. Oncol. Biol. Phys., 34(2), 481-487.

26. Young, M. E., \& Kornelsen, R. O. (1983). Dose corrections for low-density tissue inhomogeneities and air channels for 10-MV x rays. Med. Phys., 10, 450-455.

27. Lulu, B. A., \& Bjärngard, B. E. (1982). A derivation of Batho's correction factor for heterogeneities. Med. Phys., 9, 907-909. DOI: 10.1118/1.595201.

28. El-Khatib, E., \& Battista, J. J. (1984). Improved lung dose calculation using tissue-maximum ratios in the Batho correction. Med. Phys., 11(3), 279-286. DOI: 10.1118/1.595495.

29. du Plessis, F. C. P., Willemse, C. A., Lötter, M. G., \& Goedhals, L. (2001). Comparison of the Batho, ETAR and Monte Carlo dose calculation methods in CT based patient models. Med. Phys., 28(4), 582-589. DOI: $10.1118 / 1.1357223$.

30. Sontag, M. R., \& Cunningham, J. R. (1977). Corrections to absorbed dose calculations for tissue inhomogeneities. Med. Phys., 4(5), 431-436. DOI: 10.1118/1.59432930

31. Wong, J. W., \& Henkelman, R. M. (1982). Reconsideration of the power-law (Batho) equation for inhomogeneity corrections. Med. Phys., 9(4), 421-430. DOI: $10.1118 / 1.595098$.

32. Lulu, B. A., \& Bjärngard, B. E. (1982). Batho's correction factor combined with scatter summation Med. Phys., 9(3), 372-377. DOI: 10.1118/1.595174.

33. Wong, J. W., \& Purdy, J. A. (1990). On methods of inhomogeneity corrections for photon transport. Med. Phys., 17(5), 807-814. DOI: 10.1118/1.596555. 